\title{
Changes in Gut Viral and Bacterial Species Correlate with Altered 1,2-Diacylglyceride Levels and Structure in the Prefrontal Cortex in a Non-Human Primate Model of Depression.
}

\section{Jing Wu}

The First Affiliated Hospital of Chongqing Medical University

\section{Tingjia Chai}

Chongqing Medical University

\section{Hanping Zhang}

The First Affiliated Hospital of Chongqing Medical University

\section{Yu Huang}

The First Affiliated Hospital of Chongqing Medical University

\section{Seth W Perry}

State University of New York (SUNY) Upstate Medical University

\section{Yifan Li}

The First Affiliated Hospital of Chongqing Medical University

\section{Jiajia Duan}

The First Affiliated Hospital of Chongqing Medical University

\section{Xunmin Tan}

The First Affiliated Hospital of Chongqing Medical University

Xi Hu

The First Affiliated Hospital of Chongqing Medical University

Yiyun Liu

The First Affiliated Hospital of Chongqing Medical University

Juncai Pu

The First Affiliated Hospital of Chongqing Medical University

\section{Haiyang Wang}

The First Affiliated Hospital of Chongqing Medical University

Xin Jin

Stomatological Hospiral of Chongqing Medical University

Jinlin Song

Stomatological Hospital of Chongqing Medical University

Ping Ji 


\section{Peng Zheng}

The First Affiliated Hospital of Chongqing Medical University

\section{Peng Xie ( $\nabla$ xiepeng@cqmu.edu.cn)}

Chongqing Medical University https://orcid.org/0000-0002-5319-7842

\section{Research}

Keywords: major depressive disorder, Macaca fascicularis, gut microbiome, lipidomics, metagenomics Posted Date: December 31st, 2020

DOl: https://doi.org/10.21203/rs.3.rs-136570/v1

License: (c) (1) This work is licensed under a Creative Commons Attribution 4.0 International License. Read Full License 
2 Changes in gut viral and bacterial species correlate with altered 1,23 diacylglyceride levels and structure in the prefrontal cortex in a non-human 4 primate model of depression.

\section{Authors}

6 Jing $W u^{1,2,3 *}$, Tingjia Chai ${ }^{2,4 *}$, Hanping Zhang ${ }^{1,2}, Y u$ Huang ${ }^{1,2}$, Seth W. Perry ${ }^{5,6}$,

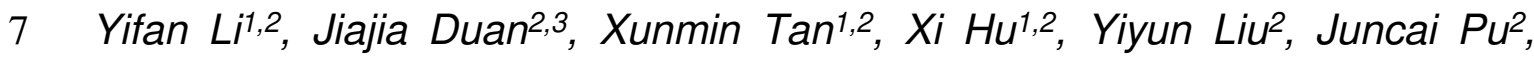
8 Haiyang Wang',7, Jinlin Song7,8, Xin Jin ${ }^{7,8}$, Ping Ji ${ }^{7,8}$, Peng Zheng',2† and Peng $9 \mathrm{Xie}^{1,2 \dagger}$

\section{Affiliations}

11 'Department of Neurology, The First Affiliated Hospital of Chongqing Medical University,

12 Chongqing, 400016, China

$13{ }^{2}$ NHC Key Laboratory of Diagnosis and Treatment on Brain Functional Diseases, The First 14 Affiliated Hospital of Chongqing Medical University, Chongqing, 400016, China

15 'The M.O.E. Key Laboratory of Laboratory Medical Diagnostics, the College of Laboratory 16 Medicine, Chongqing Medical University, Chongqing, 400016, China

$17{ }^{4}$ College of Biomedical Engineering, Chongqing Medical University, Chongqing, 400016, China 18 5Department of Psychiatry and Behavioral Sciences, College of Medicine, State University of 19 New York (SUNY) Upstate Medical University, Syracuse, NY USA

$20{ }^{6}$ Department of Neuroscience \& Physiology, College of Medicine, SUNY Upstate Medical 21 University

$22{ }^{7}$ Chongqing Key Laboratory of Oral Diseases and Biomedical Sciences, Stomatological 23 Hospital of Chongqing Medical University, 401147 Chongqing, China

$24{ }^{8}$ Key Laboratory of Psychoseomadsy, Stomatological Hospital of Chongqing Medical University 25

26 *These authors contributed equally to this study.

27 tThese authors are co-senior authors.

\section{Corresponding authors}

29 Professor Peng Xie; E-mail: xiepeng@cqmu.edu.cn

30 Professor Peng Zheng; E-mail: peng-zheng@foxmail.com 


\section{Abstract}

32 Background: Major depressive disorder (MDD) is a debilitating mental disease, but its underlying molecular mechanisms remain obscure. Gut microbiome can

34 modulate brain function and behaviors through the microbiota-gut-brain (MGB) axis in depression. Our previously established non-human primate model of naturally occurring depression-like behaviors, which is characterized by MGB axis disturbances, can be used to interrogate how a disturbed gut ecosystem may modulate the MDD onset. To better clarify the molecular interrelationships and downstream functional consequences in the MGB axis on MDD pathology, here, gut metagenomics were used to characterize how gut virus and bacterial species, and associated metabolites, change in depressive monkey model.

Results: We identified a panel of 33 gut virus and 14 bacterial species that could discriminate the depression-like (DL) from control M. fascicularis. In addition, using lipidomic analyses of central and peripheral samples obtained from these animals, we found that the DL macaque were characterized by alterations in the relative abundance, carbon-chain length, and unsaturation degree of 1,2-diacylglyceride (DG) in the prefrontal cortex (PFC), in a brain region-specific manner. In addition, lipid-reaction analysis identified more active and inactive lipid pathways in PFC than in amygdala or hippocampus, with DG being a key nodal player in these lipid pathways. Significantly, co-occurrence

51 network analysis showed that altered gut viral and bacterial species, and their 52 interaction may be relevant to the onset of negative emotions behaviors by modulating the DG levels in PFC in the depressive macaque.

54 Conclusions: Our findings suggest that altered gut virus and bacteria as well 55 as DG levels and structure in the PFC are hallmarks of the DL macaque, thus 56 providing a new framework for understanding the gut microbiome's role in 57 depression. 
Keywords: major depressive disorder, Macaca fascicularis, gut microbiome, lipidomics, metagenomics

\section{Background}

Major depressive disorder (MDD) is a serious mental disorder that imposes a substantial burden on families and society worldwide[1]. Currently, the underlying molecular basis of MDD remains uncertain. In addition, only a subset of patients with MDD are effectively treated by the currently available antidepressant drugs, which have been developed based on the existing theories for the molecular basis of $\operatorname{MDD}[2,3]$. Therefore, identification of new molecular mechanisms and more effective therapies for MDD are required.

Emerging evidence suggests that the gut microbiome can modulate brain function and behaviors through the microbiota-gut-brain axis (MGB), and plays a vital role in the pathogenesis of various mental disorders such as autism, bipolar disorder, and schizophrenia[4-7]. Recently, we and others found that patients with MDD had disturbed gut microbiomes, but the underlying mechanisms by which the gut microbiome may contribute to the pathogenesis of MDD are unclear. Previously, we established and reported a non-human primate(NHP) model of naturally occurring depression-like behaviors, which developed under conditions of chronic social dominance stress[8, 9]. This unique animal model replicates many characteristics of depression onset and behaviors in humans. This primate depression model was also characterized by MGB axis disturbances, thus providing an ideal model to investigate the gut ecosystem's roles in depression development.

Previously, integrated metabolomic and metagenomic analyses in these DL macaques found that their altered gut microbiota were associated with brainregion specific glycerophospholipid metabolism changes the prefrontal cortex 
85 (PFC), amygdala (AMY), and hippocampus (HIP)[8]. Similar alterations were also found in the PFCs of germ-free mice that received fecal microbial transplants (FMTs) from MDD subjects[10]. Gut microbiota can influence MGB lipid and fatty acid production and metabolism, and lipid peroxidation and signaling pathway activation[11]. However, further studies are needed to better clarify the molecular interrelationships and downstream functional consequences of these changes on MDD pathology.

Lipids are a group of distinct biological molecules which make up over $50 \%$ of the brain's dry mass, and are vital participants in fundamental neurobiological processes including neuronal membrane synthesis, and vesicular and synaptic transmission and trafficking. There are major subclasses of lipids such as fatty acids; glycerolipids such as mono-, di-, and tri-acylglycerol (MG, DG, and TG, respectively); and glycerophospholipids such as phosphatidylcholine (PC), phosphatidic acid (PA), and phosphatidylglycerol (PG). In the brain, with various enzymatic reaction systems, lipids achieve rapid transformations across subclasses, and disrupted lipid transformations (and thus equilibrium) can manifest as a variety of neurological diseases[12, 13]. Moreover, the

102 structures and dynamic activities of neural cells' membranes are profoundly

103 sensitive to lipid structure, including acyl carbon chain length and 104 unsaturation[14]. For neurons and glia, and their vesicles, changes in membrane permeability and fluidity, as regulated by lipid composition and

106 structure, help control synaptic excitability and transmission, and the 107 stabilization of ion channels[15, 16]. Short-chained and saturated lipids 108 generate a more tightly packed and compressed cell membrane whilst, in 109 contrast, longer-chained and unsaturated lipids occupy more space and are 110 more mobile in membranes.

111 For these reasons, here we used shotgun metagenomic analysis of fecal 112 samples, and mass spectrometry (MS) lipidomic analysis of brain regions (PFC, 
113 AMY, and HIP) and plasma, from 12 female DL macaques (Macaca. fascicularis)

114 and healthy controls $(\mathrm{HC})$. With these well-established bioinformatic 115 approaches, the differential gut virus, bacteria, and brain lipids between the DL 116 and $\mathrm{HC}$ macaques were identified. Based on metabolic reactions among lipids,

117 we identified the involved pathways and predicted the active or inactive lipid 118 reaction pathways in key brain regions involved in MDD pathology. Finally, we 119 integrated these multi-level omics findings by network analysis to connect and

120 correlate the altered virus and bacteria with the observed DL behaviors, to 121 further uncover how these disturbed signatures may modulate the host's 122 metabolism and behavioral function.

\section{Methods}

\section{Ethics Statements}

Detailed ethics approvals were shown in Declaration. In brief, this study was performed in strict accordance with the recommendations in the "Guide for the Care and Use of Laboratory Animals" of the Institute of Neuroscience at Chongqing Medical University (\#20100031). The sample sizes ( $n=6 /$ every group) were chosen based on the data of previous NHP studies [17, 18], and our previous works on behavior and biochemical measures in $M$. fascicularis[19]. Macaques were housed in an environmentally controlled facility $\left(22 \pm 1^{\circ} \mathrm{C}\right.$ temperature; $50 \pm 5 \%$ relative humidity; and $12 \mathrm{~h}$ light/12h dark cycle with lights on at 7:00 AM).

\section{Behavioral observation and tissue preparation}

We identified M. fascicularis animals with naturally occurring DL behaviors as described previously[8, 19]. Briefly, we surveyed the populations from 20 enclosures, and identified 6 typical DL and 6 HC macaques. All 12 identified macaques were adult female and disease-negative by veterinary examination. 
139 The DL behaviors Huddle, Sit alone, Locomotion, and Amicable were observed 140 and identified via "free enclosures tests"[20]; the behavior Communication was 141 evaluated and identified by "social interaction test". The "free enclosures test" 142 was conducted within the macaque's native habitat housing, via video records

143 collected for $20-30$ min per shoot, with 6-7 replicates over 3 days. The "social 144 interaction test" was performed in isolated cages, with video records collected 145 for 30 min per shoot with 2 repeats. Based on the similarity with human

146 emotions, behaviors Huddle and Sit alone were identified as negative emotions, 147 behaviors Locomotion, Amicable and Communication were identified as 148 positive emotions. All behavioral observations were videotaped and analyzed 149 by NOLDUS Observer XT software (version 10.0, Noldus Information Tech 150 Technology, Leesburg, PA). The detailed behavioral results have been 151 published previously[8, 19, 20]. Following euthanization, whole brains were 152 isolated and immediately frozen in liquid nitrogen, and cut into $5 \mathrm{~mm}$ slices along 153 the coronal plane[21, 22]. The PFC, HIP, and AMY were dissected according to 154 a macaque brain atlas $[23,24]$, then stored at $-80^{\circ} \mathrm{C}$ until use.

\section{Metagenomic analysis of fecal samples}

156 The metagenomic libraries were prepared and sequenced according to our 157 previous protocols[25, 26]. Briefly, we extracted microbial DNA from the 6 DL 158 and $6 \mathrm{HC}$ macaque fecal collections using the E.Z.N.A. Stool DNA Kit (Omega 159 Bio-tek, Norcross, GA, USA). The DNA extract was sheared using Covaris $160 \mathrm{M} 220$, aimed insert size was about $300 \mathrm{bp}$. A paired-end library was constructed 161 using TruSeqTM DNA Sample Prep Kit (Illumina). Then, the amplicons library 162 was paired-end sequenced on an Illumina HiSeq $X$ platform[27]. Threshold 163 exclusion in sequence quality control and genome assembly was performed as 164 we described previously[25, 26]. Reads were aligned to the macaque (Macaca 165 fascicularis) genome by BWA (http://bio-bwa.sourceforge.net), and any hit 
166

associated with the reads and their mated reads were removed. Metagenomics data were assembled using MEGAHIT [28](https://github.com/voutcn/megahit), contigs with the length being or over $300 \mathrm{bp}$ were selected as the final assembling result. All predicted genes with a $95 \%$ sequence identity $(90 \%$ coverage) were clustered using CD-HIT[29] (http://www.bioinformatics.org/cdhit/). Reads after quality control were mapped to the representative sequences with 95\% identity using SOAPaligner[30] (http://soap.genomics.org.cn/). Here, prior to construction of gene sets, we initially removed the host sequences with high homology to the macaca genome from microbial metagenomes. Based on the NCBI NR database, we annotated gene sets for bacteria, fungi, viruses, protozoa, and archaea using Diamond (Version 0.8.35). Based on a unified database, each gene is assigned to the highest scoring taxonomy, which facilitates simultaneous assessment of these microbial species in the gut ecosystem of depressive macaca [31]. The differential bacterial species and gut viruses between the two groups were identified using Linear discriminant analysis (LDA) Effect Size (LEfSe) with an LDA score >2.0[32].

\section{Lipid identification and analysis}

Details of the lipidomic methods were based on LC-MS/MS, similar to our previously published protocols[8, 26]. Briefly, brain tissue samples were prepared by homogenization, dissociation, and centrifugation, and plasma samples were collected and centrifuged twice ${ }^{[25]}$. Chromatographic conditions: the column is Hypersil GOLD C18 $(100 \times 2.1 \mathrm{~mm}, 1.9 \mu \mathrm{m})$; the mobile phase $A$ is acetonitrile: water $(60: 40, \mathrm{~V} / \mathrm{V})$, mobile Phase $B$ is acetonitrile: isopropanol $(10: 90, V / V)$; the flow rate was $0.40 \mathrm{~mL} / \mathrm{min}$, the injection volume was $5 \mu \mathrm{l}$, and the column temperature was $45^{\circ} \mathrm{C}$. Mass spectrometry was conducted using Thermo Scientific ${ }^{\mathrm{TM}} \mathrm{Q}$ Exactive $^{\mathrm{TM}}$ quadrupole-qrbitrap mass spectrometer.

Mass spectrometry peaks were identified using the Thermo Scientific ${ }^{\mathrm{TM}}$ 
193 LipidSearch $^{\mathrm{TM}}$, which contains over 150 million lipid species. Sample names,

194 lipid information (species, subclass, structural information), and normalized 195 peak area percentages, were integrated and imported into SIMCA-P+14.0

196 (Umetrics, Umeå, Sweden). Discrimination of lipid species between DL and HC

197 samples was analyzed and visualized using partial least squares discriminant 198 analysis (PLS-DA)[33]. To identify the differential lipid species responsible for 199 discriminating between the two groups, we used threshold of variable 200 importance plots $(\mathrm{VIP})>1.0$ with statistical significance set at $\mathrm{P}<0.05$.

201 Lipid reaction activities in lipid pathways

202 The reaction activities in lipid pathways can be predicted based on methods 203 as described previously[34, 35]. This method calculated statistical $Z$ scores for 204 all possible lipid pathways in order to predict whether a particular pathway is 205 active or inactive in $\mathrm{DL}$ as compared to HC. Reactions with higher $Z$ scores 206 were identified as active. First, we examined all possible lipid pathways from 207 Reactome (http://www.reactome.org/) and constructed a pathway network. 208 Based on the pathway network, we computed the reaction weight vector ()as a 209 ratio of product $\left(A_{i+1}\right)$ over substrate $\left(A_{i}\right)$ for each edge in the network $\left(\omega_{i}=\right.$ $\left.210\left(A_{i+1} / A_{i}\right)(i=1,2, \ldots, k-1)\right)$. Then, for each weighted edge of the pathway, one211 sided Student's t-tests were performed using the weights between DL and HC, 212 to identify reactions with differential activity. By assuming that the t-distribution 213 can be approximated by a normal distribution, the $P$ values were converted to $214 Z$ scores using the cumulative distribution function $C D F, Z=C D F^{-1}(1-P)$. We 215 chose the significance level $(P)$ to be 0.05 , corresponding to a $Z_{i}>1.645$ for a 216 reaction to be considered as significantly active. For visualization, the $Z$ scores 217 were multiplied by -1 for the reactions which were significantly more active in $218 \mathrm{HC}$ as opposed to DL. Finally, we calculated an average $Z$ score for lipid 219 pathways to detect consistent changes in the flux across multiple reaction steps. 
$220 Z$ score for pathway $\mathrm{A}\left(Z_{A}\right)$ was calculated by all $Z_{i}(i=1,2, \ldots, k-1)$ of reactions

221 in the pathway, as follows:

$$
Z_{A}=\frac{1}{\sqrt{k-1}} \sum_{i=1}^{k-1} Z_{i}
$$

As shown in references [34, 35], $Z_{A}$ also follows a normal distribution. To determine if pathway A was significantly more active, we again chose the significance level $(P)$ to be 0.05 , corresponding to a $Z_{i}>1.645$ for reaction to 226 be considered as significantly active. Again, these $Z$ scores were multiplied with $227-1$ for the reactions which were significantly more active in $\mathrm{HC}$ as opposed to DL. For visualization, a negative Z-score indicates inactive pathway in DL 229 relative to $\mathrm{HC}$, and a positive Z-score indicates active pathway in $\mathrm{DL}$ relative to $230 \mathrm{HC}$.

\section{Statistical analyses}

Statistical analyses were carried out using SPSS (version 21) and R studio (version 3.5.2, 2018), unless otherwise described. The discriminating lipid species between the two groups were considered significant at the threshold of VIP $>1.0$ and P-values $<0.05$. The levels of all lipid subclasses were analyzed by unpaired two-tailed Student's t-tests. Error bars represent the standard error of the mean (SEM) in all cases. LEfSe analysis was used to identify the different

238 gut bacteria and viruses by estimating the effect of the abundance of each 239 species (LDA > 2 and $P$ value $<0.05)$. In established lipid pathways, the reactions were considered as significantly active (or inactive) for their calculated

241 Z score $>1.645$ (or $<-1.645$ ), corresponding to the P-value $<0.05$. Correlation 242 data analysis was performed using the spearman's regression model with the 243 significance threshold of $P<0.05$. The investigators were not blinded to the 244 group classification while analyzing the data. 


\section{Gut virus differences between DL and HC macaques}

The obtained metagenomic sequencing was mapped to the known viral genomes NCBI NR database. From the filtered sequence data, we annotated 1311 viral species based on the NR database. Using LEfSe analysis, we

250 identified 33 differential gut viral species that could discriminate between the

251 DL and HC groups (Figure 1a, Supplemental Table 1). Compared to HCs, the 252 DLs were characterized by 16 enriched gut viruses, mainly belonging to the 253 Myoviridae (7 species) and Siphoviridae (4 species) families, and 17 depleted 254 viruses mainly from the Podoviridae (6 species) and Siphoviridae (6 species) 255 families. Interestingly, the majority of altered gut viruses (25/33, 75.76\%) 256 belonged to the order Caudovirales, suggesting altered Caudovirales ecology 257 was a hallmark of the DL animals.

\section{Gut bacterial species differences between DL and HC macaques}

Here, using our previously published metagenomic data[8], we reannotated 468 bacteria families and 11653 species based on NR database. Using LEfSe analysis, we identified 14 differential gut bacterial species responsible for

262 distinguishing the DL and HC groups (Figure 1b, Supplemental Table 2).

263 Compared to the HC group, the DL group was characterized by 6 increased 264 bacteria species, mainly belonging to the Paraprevotella family (3 species), and 2658 decreased species mainly from the Streptococcaceae (3 species) and 266 Gemella (3 species) families. The majority of altered bacterial species (9/14, $26764.28 \%$ ) belonged to the phylum Firmicutes.

DL macaques have different lipid composition in plasma and depression relevant brain regions

270 In total, we identified 2238, 2153, and 2074 lipid species in PFC, AMY, and 271 HIP, respectively. The proportions of lipid subtypes between brain regions were 
272 generally similar, with phosphatidylethanolamine (PE), PC, and 273 phosphatidylserines (PS) being the most abundant species in each region, 274 together comprising $67.7 \%, 68.7 \%$, and $68.2 \%$ of all lipid species in PFC, AMY, 275 and HIP respectively (Figure 2a). Lipid species found in plasma were mainly 276 comprised of PE, PC, and triacylglycerol (TG) subtypes, together comprising $27765.8 \%$ of the 1591 lipid species found in plasma (Figure 2a). PLS-DA was 278 performed to determine whether the overall lipidomic signatures of $\mathrm{DL}$ 279 macaques were significantly different from those of controls. These lipidomics 280 assays identified robust differences between HCs and DLs in PFC, AMY, HIP, 281 and plasma (Figure 2b).

\section{DL macaques have different lipid levels and structure in plasma and} depression relevant brain regions

We compared the relative abundance of each lipid subclass between DL and $\mathrm{HC}$, and found that there were specifically altered lipid subclasses in the same three brain regions. Normalizing against the corresponding lipid level in

287 HCs, we found DG was significantly increased in PFC ( $F C=1.38, P=0.0008)$

288 (Figure 3a), PA was significantly increased in AMY (FC=1.18, $P=0.04)$ (Figure 289 3b), and monosialotetrahexosylganglioside (GM1) was significantly increased 290 in HIP $(F C=1.51, P=0.04)$ (Figure 3c). Then we further identified the 291 discriminating lipid species between DL and HC macaques using double cut-off 292 (results were considered statistically significant if $\mathrm{P}<0.05$ and VIP $>1.0$ ). Of 293 the 72 total discriminating lipid species, we found that HIP had the most (28 294 species), while AMY had the least (7 species) (Supplemental Figure 1). These 295 results were consistent with what we previously reported based on untargeted 296 metabonomics[8]. 

macaques

Brain function relies on the homeostasis of cellular membranes, and its perturbation might partially explain the neuronal deficits found in depression.

301 The carbon chain length and the degree of unsaturation of fatty-acyls are two 302 key elements of lipid architecture that impact membranes' functional 303 biophysical properties. Our lipidomic analyses demonstrated not only 304 differences in relative lipid composition and levels between $\mathrm{HC}$ and DL 305 macaques, but also differences in lipids' biochemical structures with potential 306 structure-function consequences on neural cell activity. Among the altered lipid 307 subclasses, DG showed profound structural alterations, with multi-level 308 alterations of carbon chain length and degree of unsaturation. Compared with $309 \mathrm{HC}$, the DL macaques had altered levels of DGs with carbon chain lengths of $31034 \mathrm{C}, 36 \mathrm{C}$, and $43 \mathrm{C}$ in PFC, and 38C in AMY and HIP (Figure 3d). The 311 differential carbon chain length DGs were primarily in the range of $34 \mathrm{C}$ to $40 \mathrm{C}$, 312 a range which comprised the majority of DG species. We also analyzed the 313 degree of unsaturation for each subclass and DG. Compared with HCs, the 314 degree of unsaturation of DG was significantly increased in 2, 3, and 10 double 315 bones in PFC, but there was no difference in unsaturation degree of DG in AMY 316 or HIP (Figure 3e). Taken together, DG structure and degree of unsaturation 317 was the most different in the PFC, in $\mathrm{HC}$ versus DL macaques.

318 In the periphery (plasma), the structural changes in DG species were 319 distinctly different than seen in brain. In plasma, the typical DG carbon chain 320 length was longer, mainly $62 \mathrm{C}$ to $67 \mathrm{C}$ (Figure 3d). The degree of unsaturation 321 was lower, mainly 0 to 3 (Figure $3 \mathbf{e}$ ), compared with 2 to 6 carbon bones of DG 322 in brain. There were no difference in carbon chain length or unsaturation degree 323 in plasma DG. 


\section{DG-related lipid pathway activity was altered in DL macaques}

Given the altered abundance and structure of brain DG, next we employed 326 lipid-reaction analyses to explore whether specific lipid pathways were dysregulated in key depression-relevant brain regions in the DL macaque.

328 Based on the Reactome and KEGG databases, we measured each lipid reaction and mapped them into lipid pathways. Briefly, the upstream and downstream transformations of any lipid species can be viewed as an edge, that can be evaluated by statistical Z-scores for predicting active and inactive

332 pathways. Using a significance level of $P=0.05$, corresponding to $Z>1.645$

333 for a reaction to be considered significantly activated, we found altered lipid 334 pathway activity in PFC, HIP, and AMY. In the PFC of DL macaques, 3 lipid 335 pathways were significantly activated (LPC-PC-PA-DG-TG, LPC-PC-PA-LPA, 336 and LPC-LPA), and 2 lipid pathways significantly inactivated (DG-PC-LPC, DG337 PA-PI-LPI) relative to HCs (Figure 4a-c). In the HIP, the LPC-LPA pathway was activated, and the LPA-PA-PG-LPG pathway inactivated, in DL versus HC.

339 (Figure 4b, c, Addition file 2 Figure S2a). LPC-LPA activity was significantly 340 increased in both PFC and HIP (Figure 4b), but otherwise no lipid pathway 341 activity was significantly changed across more than one brain region examined 342 (Figure 4b, 4c). This most dysregulation of lipid pathway activity occurred in 343 PFC (3 active, 2 inactive, in DL relative to $\mathrm{HC}$ ), followed by HIP (one up, one 344 down) (Figure 4b, 4c).

345 We also calculated Z-scores for synthesis and degradation of DG and PA. 346 Interestingly, for $\mathrm{DL}$ relative to $\mathrm{HC}$, we found significantly activated synthesis 347 and inactivated degradation of PA in the AMY (Addition file 2 Figure S2c, S2d, 348 respectively), which corroborates the higher PA levels in DL AMY as measured 349 by MS (Figure $3 \mathbf{b}$ ). However, the accumulation of DG in the DL PFC (Figure 350 3a) appears to arise principally from inactivated degradation, although DG 351 synthesis in the DL PFC trended toward an increase but was not significant 
(Addition file 2 Figure S2c, S2d, respectively).

Next, we explored the molecular geometry of lipids in these altered 354 pathways and found some interesting lipid shape changes in these altered lipid 355 pathways in the PFC of the DL macaques. Based on the size ratio of the 356 hydrophilic head to the hydrophobic tail, glycerophospholipids and glycerolipids can be classified into cone (small head, two or more hydrophobic tails; including 358 DG, PA, and TG), cylinder (large head and two hydrophobic tails; including PC 359 and PI), or inverted cone (large head and single hydrophobic tail; including LPI 360 and LPC) shapes. As shown in Figure 4f, the altered lipid pathways tend to 361 promote (red arrow) lipid shape evolution from inverted cone lipids to cylinder 362 and cone lipids, and to inhibit (blue arrows) the opposite evolution. DG lay at 363 the intersection of these evolutions of lipid molecular geometry, and was 364 involved in multiple processes.

Co-occurrence network analysis of changes in gut viruses, bacteria, and DG levels in DL macaques versus HCs.

To explore the potential interactions of these microbiome and molecular changes along the MGB axis of DL macaques, we constructed co-occurrence networks of altered gut viruses, bacteria, and DG in the PFC of DL versus HC macaques. Using an edge-weighted spring-embedded layout, the network was

371 visualized and the nodes were spontaneously mutually attractive or exclusive 372 based on the coefficient between nodes (Figure 5). Overall, co-occurrence 373 analysis showed that gut viruses and bacteria formed strong and broad co374 occurring relationships with DG levels in PFC; and the five behavioral 375 phenotypes were divided, and the other nodes were spontaneously clustered 376 with positive covariation around the behaviors. In the left region of this 377 generated co-occurrence network, we found that two viral clusters (Myoviridae 378 (\#1), Siphoviridae (\#3)) and a bacterial cluster (Prevotellaceae (\#2)) were 
379 directly or indirectly substantially correlated with 11 DG species in PFC; 380 meanwhile, those altered DG species in PFC of DL macaque were positively 381 correlated with negative emotions behaviors (Huddle and Sit alone). In the right 382 region of this network, alternations of two viral clusters (Siphoviridae (\#6), 383 Podoviridae (\#4)) and two bacterial cluster (Gemella (\#5), Streptococcaceae 384 (\#7)) were substantially correlated with Communication and Locomotion 385 behaviors. Meanwhile, only one DG species in PFC was positively correlated 386 with the two positive emotions behaviors. In addition, there were no gut viral or 387 bacterial clusters distributed around the Amicable behavior node. Together, our 388 findings suggest that altered gut viral and bacterial species, and their interaction may be relevant to the onset of negative emotions behaviors by modulating the DG levels in PFC in the non-human primate model of depression.

\section{Discussion}

Growing evidence suggests that disturbed gut microbiome may contribute to depression pathology, but the specific mechanisms remain unclear. Here, we combined metagenomic and brain lipidomic analyses of $M$. fascicularis macaques with naturally occurring depressive behaviors. We identified 33 altered viral species mainly belonging to Myoviridae, Podoviridae, and Siphoviridae, and found 14 altered bacterial species mainly belonging to

398 Paraprevotella, Streptococcaceae, and Gemella. In the brain, we found marked 399 disturbances of DG levels and structure in the PFC of DL macaques compared 400 to controls. Moreover, lipid reaction networks identified more activated and 401 inactivated lipid pathways in PFC than in AMY or HIP, with DG being a key nodal 402 player in these PFC lipid pathways. Finally, co-occurrence analysis showed that 403 altered gut viral and bacterial species, and their interaction were correlated with 404 onset of negative emotions behaviors by modulating the DG levels in PFC.

405 Previously, the role of the gut virome has been unexplored in depression. 
406 However, importantly, we found that our DL animals had more altered gut 407 viruses than gut bacteria, suggesting that the gut virome may play a role at least 408 equivalent to that of the gut bacteriome in the pathology of depression. The 409 three differential viral families were bacteriophages associated with gut bacteria, 410 suggesting that such viruses may influence host behaviors via regulating their 411 host bacteria. In the DL macaques, the altered phages mainly parasitize 412 Proteobacteria and Firmicutes bacteria. Interestingly, we recently found similar 413 viral disturbances in MDD patients[36], in which the altered gut viruses were 414 mainly bacteriophages too. The MDD patients had increased Siphoviridae but 415 decreased Podoviridae viral family populations, which aligns with our findings 416 in the DL macaques described herein. These findings firmly establish the 417 importance of further research on gut viruses' potential role in depression, and 418 emphasize that such phage disturbances may be both a hallmark and 419 diagnostic of depression.

420 Recent clinical investigations have reported significant changes of gut 421 microbiome in patients with MDD. These clinical findings were partly 422 inconsistent due to the demographic diversity of cohorts and analytical 423 approaches. Our NHP depressive model used herein provided an ideal model 424 to avoid those confounding factors, due to the native social structure and habitat 425 that are characteristic of this model. Like many other reports of the depression 426 microbiome[25], we again identified disturbances of the phylum Firmicutes. The 427 enriched microbiota in the DL macaques mainly belonged to the family 428 Paraprevotella, and the depleted species mainly belonging to the genera 429 Gemella and Streptococcus. Paraprevotella has been suggested as a 430 biomarker in depression[37, 38] and attention deficit hyperactivity disorder[39], 431 as well as a potential factor to inhibit plasma acetate levels and intrarenal RAS 432 activation[40]. Streptococcus strains such as Streptococcus salivarius and 433 Streptococcus thermophilus, previously recognized as pathogens, have 
434 recently been used as psychobiotics in mental health [41-43]. In our study, we

435 also found some Streptococcus enriched in healthy controls, suggesting that 436 gut Streptococci may play protective roles in the MGB axis.

437 Brain is particularly enriched in lipids, with a diverse lipid composition 438 compared to other tissues[44]. Changes in the composition and structure of 439 lipids in the brain profoundly affect neurodevelopment and signal transduction 440 in perception and emotional behavior, which may lead to depression and 441 anxiety disorders[45-47]. Previous lipidomic studies mainly focused on the 442 amount of the different lipid species, which is reasonable but there are still some 443 limitations: the variation of lipid structure and abundance. Recent studies have 444 tried to scrutinize the structural and biotransformational alteration of lipids in 445 disease. In our study, using a comprehensive approach, we tried to identify a 446 key lipid group along the MGB axis that may be relevant to depression. DG in 447 PFC was identified based on 4 aspects: first, the abundance of DG was 448 significantly higher in PFC; second, the carbon chain length and unsaturation 449 of DG were altered in at multiple levels; third, DG pathway activity was 450 profoundly altered in PFC; last, DG in PFC was deeply involved in the MGB 451 axis-behavior network.

452 In our previous studies and other preclinical and clinical experiments, 453 disturbances of glycerolipid and glycerophospholipid metabolism were 454 considered hallmarks in depression[48-51], but their role in depression 455 pathogenesis is not explicitly clear. Here, using lipidomic approaches, we 456 further showed alterations in DG pathway lipid shapes especially in the PFC of 457 DL macaques. Both DG-related reaction pathways showed shape 458 transformations from inverted cone and cylinder to cone via decreased 459 headgroup size and more hydrophobic tails. Previous studies have reported 460 that changes in bilayer curvature during vesicle fusion/fission relies on lipid 461 shapes via CHOL translocations (chains)[14, 52, 53]. The cone shaped lipids, 
462 mainly $\mathrm{PA}$ and DG, promote negative membrane curvature via various 463 phospholipases, while lyso-phosphatidylcholine (LPC) has only a single 464 hydrocarbon tail that promotes positive membrane curvature[53]. Secondly, 465 emerging evidences shows that the fatty acid chain length and unsaturation are 466 involved in anxiety and cognitive disorders by modulating membrane fluidity[54, 467 55]. Here we found that the unsaturation degree of DG in PFC was altered, and 468 the di- and tri-unsaturated DG significantly decreased, suggesting that the low 469 unsaturated fatty acids may weaken the protection of poly-unsaturated fatty 470 acids (PUFAs) in depression. As Levental et al. recently reported[56], 471 exogenous PUFAs such as docosahexaenoic acid (DHA) and $\omega-6$ arachidonic 472 acid (AA) can reduce the di- and tri-unsaturated lipid species by counteracting 473 cell membrane perturbations. Together, these findings may extend our 474 understanding of brain lipids on depression.

475 It is widely accepted that gut microbiota can modulate hosts behaviors via 476 the MGB-axis. Here we also confirmed that robust network correlations occur 477 amongst the integrated gut virus, gut bacteria, and DG changes in the PFC of 478 DL macaques, and host DL behavioral phenotypes. Interestingly, virus and 479 bacteria were spontaneously clustered, and those clusters as well as DGs 480 surrounded different kinds of behaviors, suggesting the potential modulation 481 between viruses, bacteria, and depressive behaviors. Other studies have 482 shown phages interfere with host bacteria. For example, Loeffler et al. found 483 that Podoviridae phage C1 can kill A, C, and E streptococci via lytic 484 enzymes[57]. Romero et al. further confirmed that Siphoviridae phiHER can kill 485 Streptococcus pneumoniae by specifically cleaving covalent bonds of cell wall 486 peptidoglycan[58]. In line with these findings, in our results the Podoviridae (\#4) 487 and Siphoviridae (\#6) virus clusters co-localized with the bacterial cluster 488 Streptococcaceae (\#7). These studies confirm that gut microbiota can modulate 489 the host lipid metabolism in various ways, which may offer new therapeutic 
avenues for depression.

Interestingly, the five behavioral phenotypes of our DL macaque were spontaneously separated: Huddle and Sit alone, which can represent negative emotions and unhealthy affect in macaque, co-localized with the majority of DG species, and with 3 microbial clusters; Locomotion and Communication, which can represent positive emotions and healthy affect in macaque, were colocalized with only 1 species of DG and 4 microbial clusters; but Amicable, another positive emotional behavior, located alone. These findings confirmed the reliability of our previously established behavior spectrum, and strongly suggested that the gut microbiota and brain lipids may modulate positive or negative emotion in different ways in depression. In our findings, DG mainly correlated with negative emotions rather than with positive emotions, suggesting that functional behavioral disturbances caused by changes in DG species composition, levels, and/or structure may serve to regulate or exacerbate negative emotions in depression. In contrast, positive emotions in depression may be modulated by the other unknown lipid groups. Interestingly, the unique DG species (DG (22:6/22:6)) that co-localized around positive emotions in our network analysis, is made up with a well-known fatty aciddocosahexaenoic acid (DHA). Many studies have investigated DHA supplementation as a potential treatment or prophylatic for depression. Van der Burg et al. found that DHA concentrations in red blood cell membranes were significantly correlated with a decrease in depressive symptoms during active treatment, and increased in response to depression treatment[59]. Weiser et al. fed pregnant rats with diets sufficient or deficient in DHA during gestation and lactation, and found that depressive-like behavior and its associated biomarkers in DHA-deficient offspring were worse compared with animals with sufficient levels of DHA[60]. In both these studies, the fatty acids were ingested, meaning that gut microbes would have participated in the absorption of these 
518 dietary fatty acid supplements. Based on our and these results, further studies

519 of the intertwined roles of the gut microbiome and lipids in the pathology and 520 pathogenesis of depression are warranted.

$521 \quad$ Nonetheless, there are some limitations of our study, which can also 522 provide direction for future research. First, due to the low reproductive rate and 523 morbidity, as well as ethical considerations, the sample sizes were relatively 524 small, thus the reliability of the association reported may be impacted. Second, 525 the effects on key viruses, bacteria, and brain lipids that we reported in this 526 study need further longitudinal independent validations with larger samples. 527 The key viruses and bacteria need further isolation and culturing from macaque 528 fecal samples, and more independent verifications in multiple animal models, 529 such as fecal microbiota transplantation (FMT) or microbial agents. Third, 530 depression as a complex mental disorder, and depression pathology is not 531 isolated to only the PFC, HIP, and AMY regions studied here (although those 532 are key regions of depression neuropathology). Thus further studies with 533 broader brain regions and technologies such as FMRI, calcium imaging, and 534 optogenetics are needed. Fourth, MGB crosstalk in depression involves 535 multiple mechanisms and metabolic pathways beyond those which we focused 536 on in this study. The vagus nerve, hypothalamic-pituitary-adrenal axis, and 537 neuroimmune mechanisms, are all worthy of further study.

\section{Conclusions}

539 Taken together, using metagenomic data, we found the altered gut virome, 540 especially bacteriophages, plays a role in the onset of depression. Through 541 multiomics approaches, we have presented evidence that DL macaques were 542 characterized by disturbances of gut-virus, bacteria, and DGs in the PFC. 543 Moreover, we found that disturbances of gut microbiome may be relevant to the 544 onset of negative emotions behaviors by modulating the DG levels in the 
depressive model. Our findings provide new directions to uncover the

546 pathogenesis of depression.

\section{Abbreviations}

548 MDD: Major depressive disorder; DL: Depression-like; HC: healthy controls; PFC: Prefrontal 549 cortex; NHP: Non-human primate; MGB axis: Microbial gut - brain axis; AMY: Amygdala; HIP: 550 Hippocampus; FMTs: Fecal microbial transplants; MS: Mass spectrometry; LDA: Linear 551 discriminant analysis; LEfSe: Linear discriminant analysis Effect Size; VIP: Variable importance 552 plots; SEM: Standard error of the mean; PC: Phosphatidylcholine; PE: 553 Phosphatidylethanolamine; Cer: Ceramide; LPE: Lyso-Phosphatidylethanolamine; PA: 554 Phosphatidic acids; PG: Phosphatidylglycerols; PI: Phosphatidylinositols; PS: 555 Phosphatidylserines; SM: Sphingmyelin; DG: 1,2-Diacylglycerol; LPC: 2556 Lysophosphatidylcholine; So: Sphingosine; TG: Triacylglycerol; DHA: Docosahexaenoic acid; 557 AA: $\omega-6$ arachidonic acid

\section{Declarations}

\section{Ethics approval and consent to participate}

561 This study was performed in strict accordance with the recommendations in the "Guide for the 562 Care and Use of Laboratory Animals" of the Institute of Neuroscience at Chongqing Medical 563 University (\#20100031). All work involving NHPs was conducted in accordance with the NIH 564 guide for the care and use of laboratory animals 565 (https://www.ncbi.nlm.nih.gov/books/NBK54050/) and with the recommendations of the 566 Weatherall report, "The use of non-human primates in research"[61]. We also followed nc3r 567 recommendations (https://www.nc3rs.org.uk/) by using the minimum number of depressed 568 macaques and age-matched controls, while maintaining statistical reliability. The sample sizes 569 ( $n=6 /$ every group) were chosen based on the data of previous NHP studies [17, 18], and our 570 previous works on behavior and biochemical measures in $M$. fascicularis[19]. The $M$. 571 fascicularis facilities, housing, and primate laboratories used in this study are accredited by the 572 Association for Assessment and Accreditation of Laboratory Animal Care. Macaques were 573 housed in an environmentally controlled facility $\left(22 \pm 1^{\circ} \mathrm{C}\right.$ temperature; $50 \pm 5 \%$ relative 574 humidity; and 12h light/12h dark cycle with lights on at 7:00 AM). This study does not involve 575 the use of human subjects.

\section{Acknowledgements}

578 Not applicable.

\section{$580 \quad$ Funding}

581 This work was supported by the National Key R\&D Program of China (2017YFA0505700 and 
2016YFC1307200), Non-profit Central Research Institute Fund of Chinese Academy of Medical Sciences (2019PT320002), Projects of International Cooperation and Exchanges NSFC (81820108015), the Natural Science Foundation Project of China (81971296, 81771490, 81371310, and 81200899), and Chongqing Science \& Technology Commission (cstc 2019 jcyjjqX0009).

\section{Author Contributions}

Designed the experiments: P.X. and P.Z. Performed the metagenomic analysis: J.W., T.J.C., H.P.Z., J.L.S., X.J., P.J. and Y.F.L. Performed the lipidomic analysis: J.W., T.J.C., Y.H., and J.J.D. Analyzed the lipidomic and metagenomic data: J.W., X.M.T., J.C.P and X.H. Animal behaviors: J.W., Y.Y.L, and Y.H. Drafted the manuscript: P.X, and P.Z. Revised the manuscript for intellectual content: P.X., S.W.P., and P.Z..

\section{Competing interests}

The authors have declared no conflict of interest in the submission of this manuscript.

\section{Availability of data and materials}

The metagenomics data of macaque that support the findings of this study are available from The First Affiliate Hospital of Chongqing Medical University and have published previously [8]. All the lipidomics data analysed during this study are included in this published article and supplementary information files.

\section{Consent for publication}

Not applicable.

\section{Competing interests}

Not applicable.

\section{FIGURES}

\section{Fig.1 The virus and bacteria that discriminate DL from HC groups}

LEfSe of bacteria and virus from phylum to species level. Linear discriminant analysis (LDA) combined with effect size measurements revealed a list of features that enable discrimination between the $\mathrm{HC}$ and $\mathrm{DL}$ groups in the fecal samples. a. At the viral level, the DL subjects showed 16 enriched species, mainly belonging to families Myoviridae (7 species) and Siphoviridae (4 species), and 17 depleted species mainly from the families Podoviridae (6 species) and Siphoviridae (6 species). b. At the bacterial level, the DL subjects showed 6 enriched species, mainly belonging to family Paraprevotella ( 3 species), and 8 depleted species mainly from the families Streptococcaceae ( 3 species) and Gemella ( 3 species). The discriminative variants (gut virus, bacteria species) were identified based on LDA score > 2.5. Sample set: $H C, n=6 ; D L, n=6$.

\section{Fig.2 Profile of lipidomic changes in brain and plasma.}


a. Composition of subclasses of total identified lipids in prefrontal cortex (PFC), amygdala (AMY), hippocampus (HIP) and plasma. Identified lipids exhibited similar composition of subclasses in 3 brain regions (2238, 2153, 2074, respectively). b. Partial Least Squares Discriminant Analysis (PLS-DA) showed that the PFC, HIP, AMY and plasma metabolic signatures of $D L$ group were substantially different from that in the HC group $(n=6, H C$, blue dots; $n=6$, DL, red dots). PC, Phosphatidylcholine; PE, Phosphatidylethanolamine; Cer, ceramide; LPE, lyso-Phosphatidylethanolamine; PA, phosphatidic acids; PG, phosphatidylglycerols; PI, phosphatidylinositols; PS, phosphatidylserines; SM, sphingmyelin; DG, 1,2-Diacylglycerol; LPC, 2-Lysophosphatidylcholine; So, sphingosine; TG, Triacylglycerol.

Fig.3 Significant changes exhibited in abundance and fatty acid composition of 1,2Diacylglycerol (DG) in the PFC of DL macaques.

(a-c) The abundance of some lipidomic subclasses showed significantly changes with specific brain regions, displaying the increased DG in PFC, increased PA in AMY and increased GM1 in HIP in the DL group relative to HC group. The abundance of lipidomic subclasses in DL (red bars) were normalized by whose concentrations in $\mathrm{HC}$ group (blue bars). (d-e) Analysis of fatty acyl composition of DG species by total carbon chain length and total degree of unsaturation showed variation by brain region. The carbon chain length and unsaturation degree of DG ranged congruously in three brain regions, $34-40$ and 2-5, respectively. The carbon chain length of DG altered in PFC, AMY and HIP; the unsaturation of DG altered in PFC $n=6$ per group. * $P$ $<0.05,{ }^{* *} \mathrm{P}<0.01,{ }^{* \star *} \mathrm{P}<0.001$, two-sided Student T-test; bars and points show mean \pm SEM.

Fig.4 DG-related lipid pathway activity was particularly altered in the PFC of DL macaque. Analysis of lipid pathway activity showed altered predicted lipid fluxes occurred in brain regions. Calculated $Z$-scores were chosen to indicate the active/inactive pathways in DL relative to $H C$, with the positive/negative values.(a) The PFC of DL macaque showed major altered lipid pathways, which were 3 active pathways LPC-PC-PA-DG-TG, LPC-PC-PA-LPA and LPC-LPA, and 2 inactive pathway DG-PC-LPC, DG-PA-PI-LPI, respectively. Altered lipid pathway activity was calculated by $Z$-score of each lipid classes in $\mathrm{DL}(\mathrm{n}=6)$ relative to $\mathrm{HC}(\mathrm{n}=6)$. Red and blue arrows show reactions with positive and negative activity, respectively. Colored circles indicate the fold changes in lipid abundance between 2 groups (-0.47-1.0). Deep arrows indicate the shared lipid flux across the network. (b-c) The HIP of DL macaque showed minor altered lipid pathways, including active pathway LPC-LPA and inactive pathway LPA-PA-PG-LPG. (d) molecular geometric alteration of DG related lipid pathways. Blue and yellow backgrounds indicated head groups and hydrophobic part. Dashed frames showed the geometry of lipids molecule, which can be defined as cone (DG, PA, TG), cylinder (PC and PI) and inverted cone (LPI and LPC).

Fig.5 Network analyses of changed microbiome, DG in PFC and host phenotype interactions. The microbial species changed in DL were identified by LDA (LDA > 2), including14 bacterial and 33 viral species. Host-microbiota interaction network was built from Spearman's non-parametric rank correlation coefficient $(P<0.05)$, and showed with 
coefficient $>0.70$ or $<-0.70$. Blue circles and $V$ triangles indicate the altered bacteria and virus respectively, green rhombus indicate the DG species in PFC and red rhombus indicated the depressive behaviors. Edge-weighted Spring-Embedded Layout was used to cluster nodes in accordance with coefficient. In result, there were 3 clusters correlated with the behaviors that represent negative emotions and unhealthy effects (Huddle and Sit alone), mainly focused on bacterial family Prevotellaceae and viral families Myoviridae and Siphoviridae. There were 4 clusters correlated with behaviors that represent positive and healthy emotions (Locomotion and Communication), mainly focused on bacterial families Gemella and Streptococcaceae and viral families Podoviridae and Siphoviridae. No clusters correlated with Amicable behavior that represents positive and healthy emotions. Red and blue lines indicate the correlation coefficient and the color depth was consistent with the coefficient $(-0.70-0.70)$.

\section{Supplementary information}

679 Addition file 1: Figure S1. Discriminating lipids in brain regions and plasma between $\mathrm{HC}$ and 680 DL groups.

681 Addition file 2: Figure S2. Pathway activity of lipid showed changed reactions among brain 682 regions in $\mathrm{DL}$ group relative to $\mathrm{HC}$ group.

683 Addition file 3: Table S1. Discriminatory gut virus between DL and HC groups.

684 Addition file 3: Table S2. Discriminatory bacterial species between DL and HC groups.

\section{REFERENCES}

687 1. Frankish H, Boyce N, Horton R. Mental health for all: a global goal. The Lancet. 2018;392:14936881494.

689 2. Warden D, Rush AJ, Trivedi MH, Fava M, Wisniewski SR. The STAR*D Project results: a 690 comprehensive review of findings. Curr Psychiatry Rep. 2007;9:449-459.

691 3. Ruelaz AR: Treatment-resistant depression: Strategies for management. 2006.

692 4. Sampson TR, Debelius JW, Thron T, Janssen S, Shastri GG, Ilhan ZE, et al. Gut Microbiota 693 Regulate Motor Deficits and Neuroinflammation in a Model of Parkinson's Disease. Cell. 2016;167:1469-1480 e1412.

695 5. Hsiao EY, McBride SW, Hsien S, Sharon G, Hyde ER, McCue T, et al. The microbiota modulates gut physiology and behavioral abnormalities associated with autism. Cell. 2013;155:1451.

697 6. Hu S, Li A, Huang T, Lai J, Li J, Sublette ME, et al. Gut Microbiota Changes in Patients with Bipolar Depression. Adv Sci (Weinh). 2019;6:1900752.

699 7. Zheng P, Zeng B, Liu M, Chen J, Pan J, Han Y, et al. The gut microbiome from patients with 700 schizophrenia modulates the glutamate-glutamine-GABA cycle and schizophrenia-relevant behaviors in mice. Science advances. 2019;5:eaau8317.

706 10. Chen JJ, Xie J, Zeng BH, Li WW, Bai SJ, Zhou C, et al. Absence of gut microbiota affects lipid 
metabolism in the prefrontal cortex of mice. Neurol Res. 2019;41:1104-1112.

708 11. Nicholson JK, Holmes E, Kinross J, Burcelin R, Gibson G, Jia W, et al. Host-gut microbiota metabolic interactions. Science. 2012;336:1262-1267.

12. Zhu L, Zhong M, Elder GA, Sano M, Holtzman DM, Gandy S, et al. Phospholipid dysregulation

13. Kurian MA, Meyer E, Vassallo G, Morgan NV, Prakash N, Pasha S, et al. Phospholipase C beta 1

14. Lauwers E, Goodchild R, Verstreken P. Membrane Lipids in Presynaptic Function and Disease.

15. Davletov B, Montecucco C. Lipid function at synapses. Curr Opin Neurobiol. 2010;20:543-549. Puchkov D, Haucke V. Greasing the synaptic vesicle cycle by membrane lipids. Trends Cell Biol.

18. Chu X. Preliminary validation of natural depression in macaques with acute treatments of the

19. Li X, Xu F, Xie L, Ji Y, Cheng K, Zhou Q, et al. Depression-Like Behavioral Phenotypes by Social

20. Xu F, Wu Q, Xie L, Gong W, Zhang J, Zheng P, et al. Macaques exhibit a naturally-occurring

21. Dijkman K, Sombroek CC, Vervenne RAW, Hofman SO, Boot C, Remarque EJ, et al. Prevention

22. Sorrells SF, Paredes MF, Cebrian-Silla A, Sandoval K, Qi D, Kelley KW, et al. Human hippocampal

23. Saleem KS, Logothetis NK: A combined MRI and histology atlas of the rhesus monkey brain in stereotaxic coordinates. Academic Press; 2012. NeuroMaps. [http://braininfo.rprc.washington.edu/], 2016, Accessed on Date 2016 neurogenesis drops sharply in children to undetectable levels in adults. Nature. 2018;555:377depressive-like behaviors through a pathway mediated by the host's metabolism. Mol Psychiatry. 2016;21:786-796. Zheng P, Zeng B, Liu M, Chen J, Pan J, Han Y, et al. The gut microbiome from patients with schizophrenia modulates the glutamate-glutamine-GABA cycle and schizophrenia-relevant behaviors in mice. Sci Adv. 2019;5:eaau8317.

27. Ugurel S, Schrama D, Keller G, Schadendorf D, Brocker EB, Houben R, et al. Impact of the CCR5 gene polymorphism on the survival of metastatic melanoma patients receiving immunotherapy. Cancer Immunol Immunother. 2008;57:685-691.

28. Li D, Liu C-M, Luo R, Sadakane K, Lam T-W. MEGAHIT: an ultra-fast single-node solution for large 
and complex metagenomics assembly via succinct de Bruijn graph. Bioinformatics. 2015;31:1674-1676.

751 29. Fu L, Niu B, Zhu Z, Wu S, Li W. CD-HIT: accelerated for clustering the next-generation sequencing data. Bioinformatics. 2012;28:3150-3152.

30. Li R, Li Y, Kristiansen K, Wang J. SOAP: short oligonucleotide alignment program. Bioinformatics. 2008;24:713-714.

31. Altschul SF, Madden TL, Schäffer AA, Zhang J, Zhang Z, Miller W, et al. Gapped BLAST and PSIBLAST: a new generation of protein database search programs. Nucleic acids research. 1997;25:3389-3402.

32. Zhang D, Jing X, Yang J: Biometric image discrimination technologies. IGI Global; 2006.

33. Bradley W, Steven H, Robert P. Utilities for quantifying separation in PCA/PLS-DA scores plots.

34. Nguyen A, Rudge SA, Zhang $Q$, Wakelam MJ. Using lipidomics analysis to determine signalling

35. Hahn O, Drews LF, Nguyen A, Tatsuta T, Gkioni L, Hendrich O, et al. A nutritional memory effect

36. Yang J, Zheng P, Li Y, Wu J, Tan X, Zhou J, et al. Landscapes of bacterial and metabolic signatures

37. Barandouzi ZA, Starkweather AR, Henderson WA, Gyamfi A, Cong XS. Altered Composition of

38. Liskiewicz P, Kaczmarczyk M, Misiak B, Wronski M, Baba-Kubis A, Skonieczna-Zydecka K, et al.

39. Wan L, Ge WR, Zhang S, Sun YL, Wang B, Yang G. Case-Control Study of the Effects of Gut

40. Lu CC, Hu ZB, Wang R, Hong ZH, Lu J, Chen PP, et al. Gut microbiota dysbiosis-induced activation

41. Colica C, Avolio E, Bollero P, Costa de Miranda R, Ferraro S, Sinibaldi Salimei P, et al. Evidences of a New Psychobiotic Formulation on Body Composition and Anxiety. Mediators Inflamm.

42. Simren M, Ohman L, Olsson J, Svensson U, Ohlson K, Posserud I, et al. Clinical trial: the effects

43. Marcos A, Warnberg J, Nova E, Gomez S, Alvarez A, Alvarez R, et al. The effect of milk fermented Neuron. 2015;85:695-702. 
45. Yadav RS, Tiwari NK. Lipid integration in neurodegeneration: an overview of Alzheimer's disease. Mol Neurobiol. 2014;50:168-176.

46. Kornhuber J, Rhein C, Müller CP, Mühle C. Secretory sphingomyelinase in health and disease.

47. Adibhatla RM, Hatcher JF. Phospholipase A(2), reactive oxygen species, and lipid peroxidation

48. Liu X, Li J, Zheng P, Zhao X, Zhou C, Hu C, et al. Plasma lipidomics reveals potential lipid markers

49. Liu X, Zheng P, Zhao X, Zhang Y, Hu C, Li J, et al. Discovery and validation of plasma biomarkers

50. Zheng P, Gao HC, Li Q, Shao WH, Zhang ML, Cheng K, et al. Plasma metabonomics as a novel

51. Jia HM, Li Q, Zhou C, Yu M, Yang Y, Zhang HW, et al. Chronic unpredictive mild stress leads to

52. Kulig W, Korolainen $\mathrm{H}$, Zatorska $\mathrm{M}$, Kwolek $\mathrm{U}$, Wydro P, Kepczynski $\mathrm{M}$, et al. Complex Behavior

53. Postila PA, Rog T. A Perspective: Active Role of Lipids in Neurotransmitter Dynamics. Mol

54. Oliveira TG, Chan RB, Bravo FV, Miranda A, Silva RR, Zhou B, et al. The impact of chronic stress

55. Sliz E, Shin J, Syme C, Black S, Seshadri S, Paus T, et al. Thickness of the cerebral cortex shows

56. Levental KR, Malmberg E, Symons JL, Fan YY, Chapkin RS, Ernst R, et al. Lipidomic and

57. Loeffler JM, Nelson D, Fischetti VA. Rapid killing of Streptococcus pneumoniae with a

58. Romero P, Lopez R, Garcia E. Characterization of LytA-like N-acetylmuramoyl-L-alanine

59. van der Burg KP, Cribb L, Firth J, Karmacoska D, Mischoulon D, Byrne GJ, et al. EPA and DHA as

60. Weiser MJ, Wynalda K, Salem N, Jr., Butt CM. Dietary DHA during development affects 


\section{Figures}

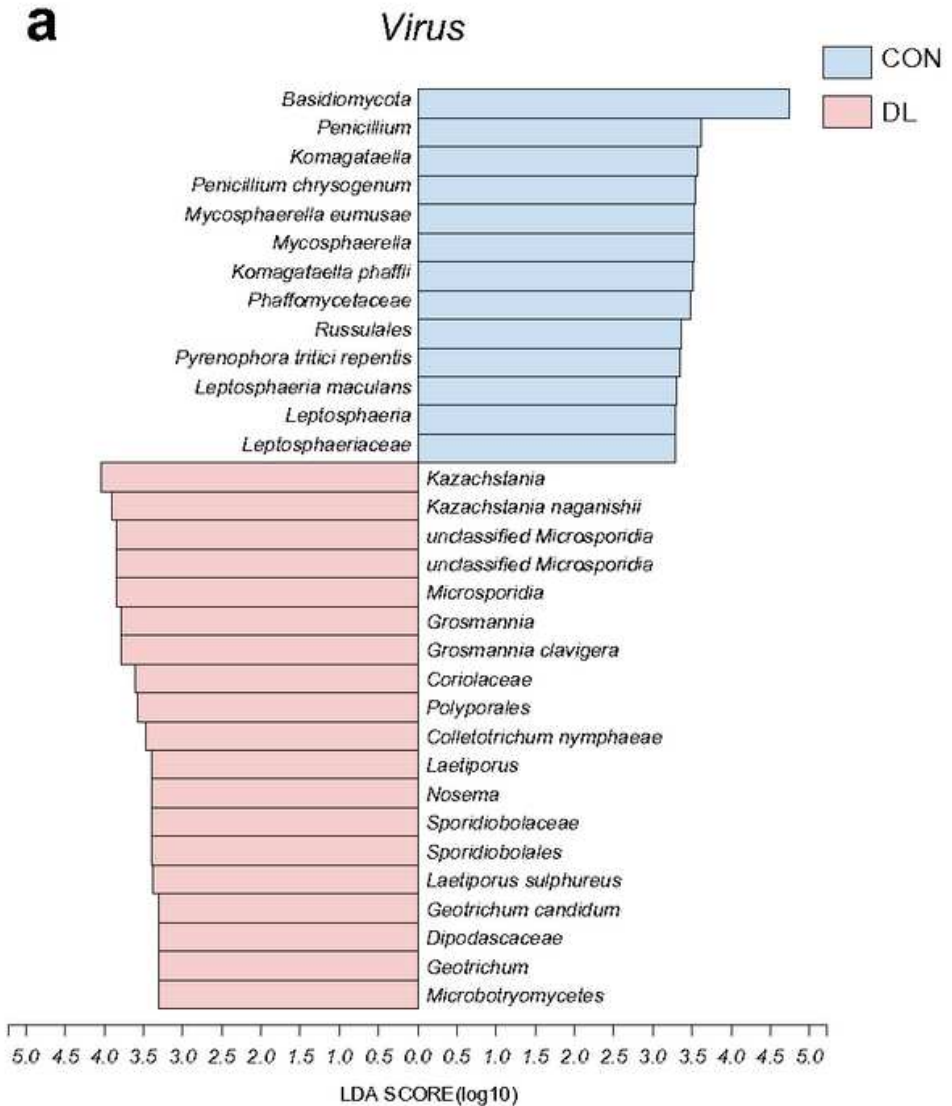

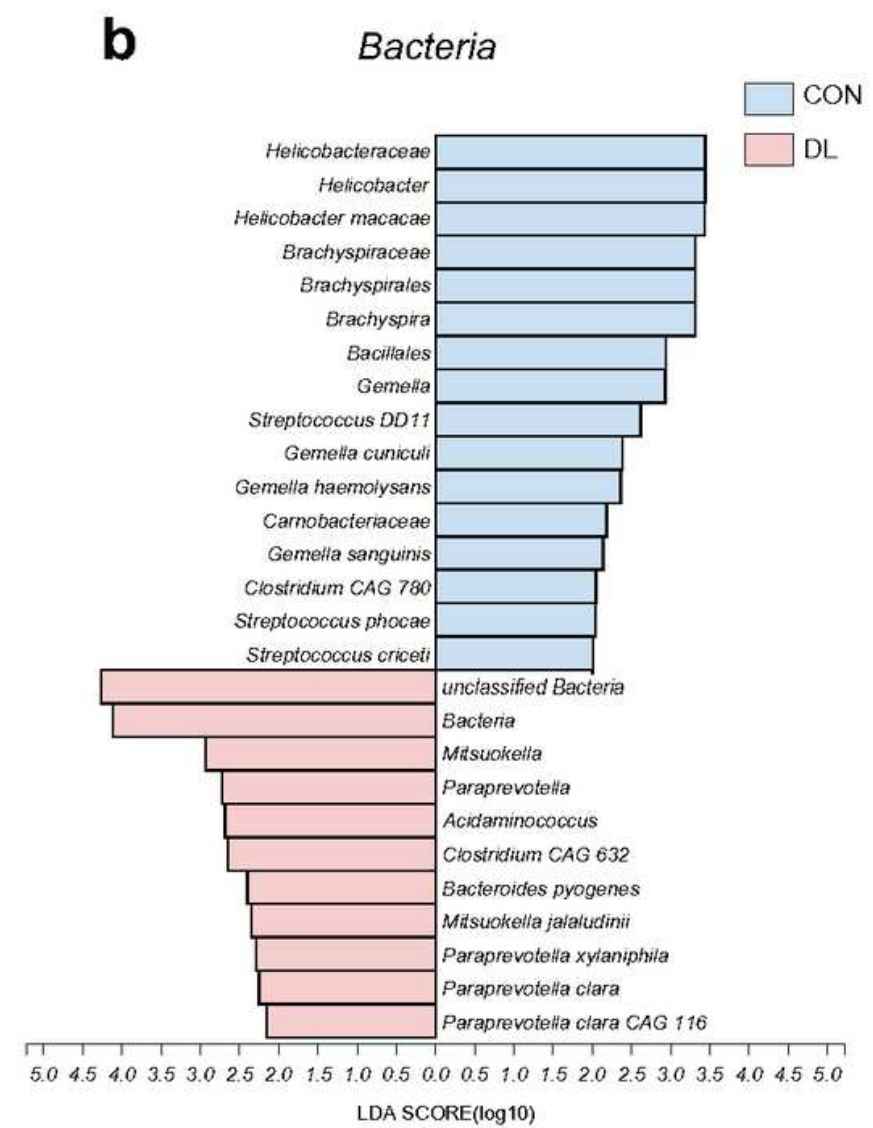

\section{Figure 1}

The virus and bacteria that discriminate DL from HC groups LEfSe of bacteria and virus from phylum to species level. Linear discriminant analysis (LDA) combined with effect size measurements revealed a list of features that enable discrimination between the $\mathrm{HC}$ and $\mathrm{DL}$ groups in the fecal samples. a. At the viral level, the DL subjects showed 16 enriched species, mainly belonging to families Myoviridae (7 species) and Siphoviridae (4 species), and 17 depleted species mainly from the families Podoviridae ( 6 species) and Siphoviridae ( 6 species). b. At the bacterial level, the DL subjects showed 6 enriched species, mainly belonging to family Paraprevotella (3 species), and 8 depleted species mainly from the families Streptococcaceae (3 species) and Gemella (3 species). The discriminative variants (gut virus, bacteria species) were identified based on LDA score $>2.5$. Sample set: $H C, n=6 ; D L, n=6$. 

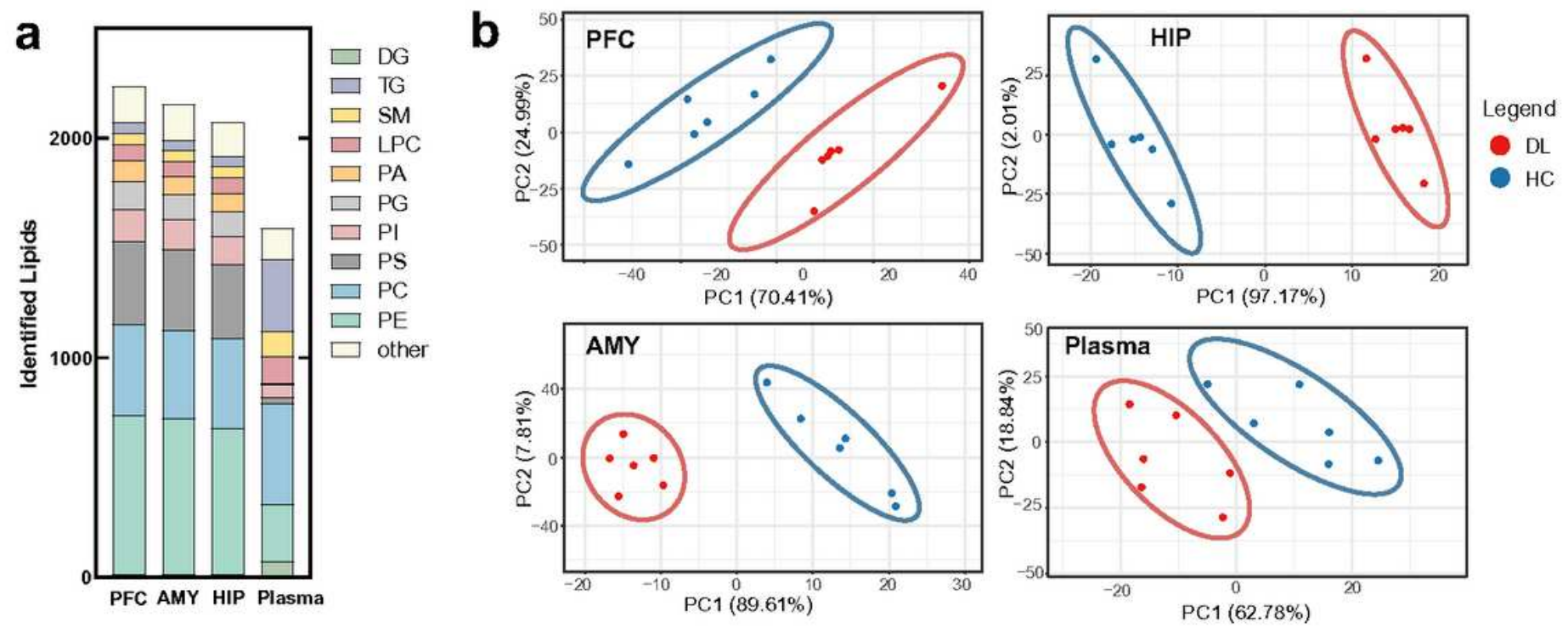

\section{Figure 2}

Profile of lipidomic changes in brain and plasma. a. Composition of subclasses of total identified lipids in prefrontal cortex (PFC), amygdala (AMY), hippocampus (HIP) and plasma. Identified lipids exhibited similar composition of subclasses in 3 brain regions $(2238,2153,2074$, respectively). b. Partial Least Squares Discriminant Analysis (PLS-DA) showed that the PFC, HIP, AMY and plasma metabolic signatures of $D L$ group were substantially different from that in the HC group $(n=6, H C$, blue dots; $n=6, D L$, red dots). PC, Phosphatidylcholine; PE, Phosphatidylethanolamine; Cer, ceramide; LPE, lysoPhosphatidylethanolamine; PA, phosphatidic acids; PG, phosphatidylglycerols; PI, phosphatidylinositols; PS, phosphatidylserines; SM, sphingmyelin; DG, 1,2-Diacylglycerol; LPC, 2-Lysophosphatidylcholine; So, sphingosine; TG, Triacylglycerol. 

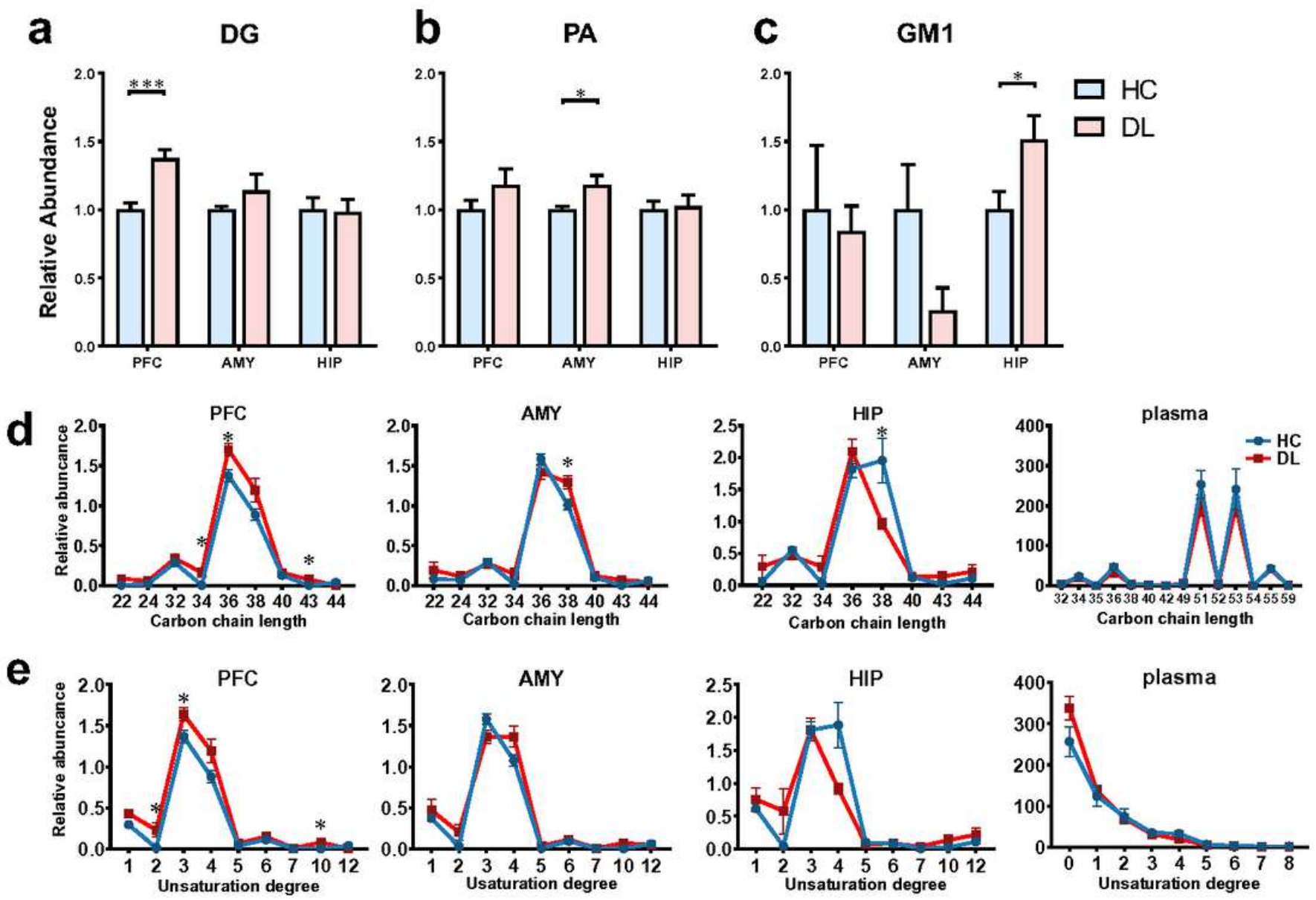

Figure 3

Significant changes exhibited in abundance and fatty acid composition of 1,2-Diacylglycerol (DG) in the PFC of DL macaques. (a-c) The abundance of some lipidomic subclasses showed significantly changes with specific brain regions, displaying the increased DG in PFC, increased PA in AMY and increased GM1 in HIP in the DL group relative to HC group. The abundance of lipidomic subclasses in DL (red bars) were normalized by whose concentrations in $\mathrm{HC}$ group (blue bars). (d-e) Analysis of fatty acyl composition of DG species by total carbon chain length and total degree of unsaturation showed variation by brain region. The carbon chain length and unsaturation degree of DG ranged congruously in three brain regions, 34-40 and 2-5, respectively. The carbon chain length of DG altered in PFC, AMY and HIP; the unsaturation of DG altered in PFC $n=6$ per group. ${ }^{\star} P<0.05$, ${ }^{\star *} P<0.01$, $* \star * P<0.001$, two-sided Student Ttest; bars and points show mean \pm SEM. 



C
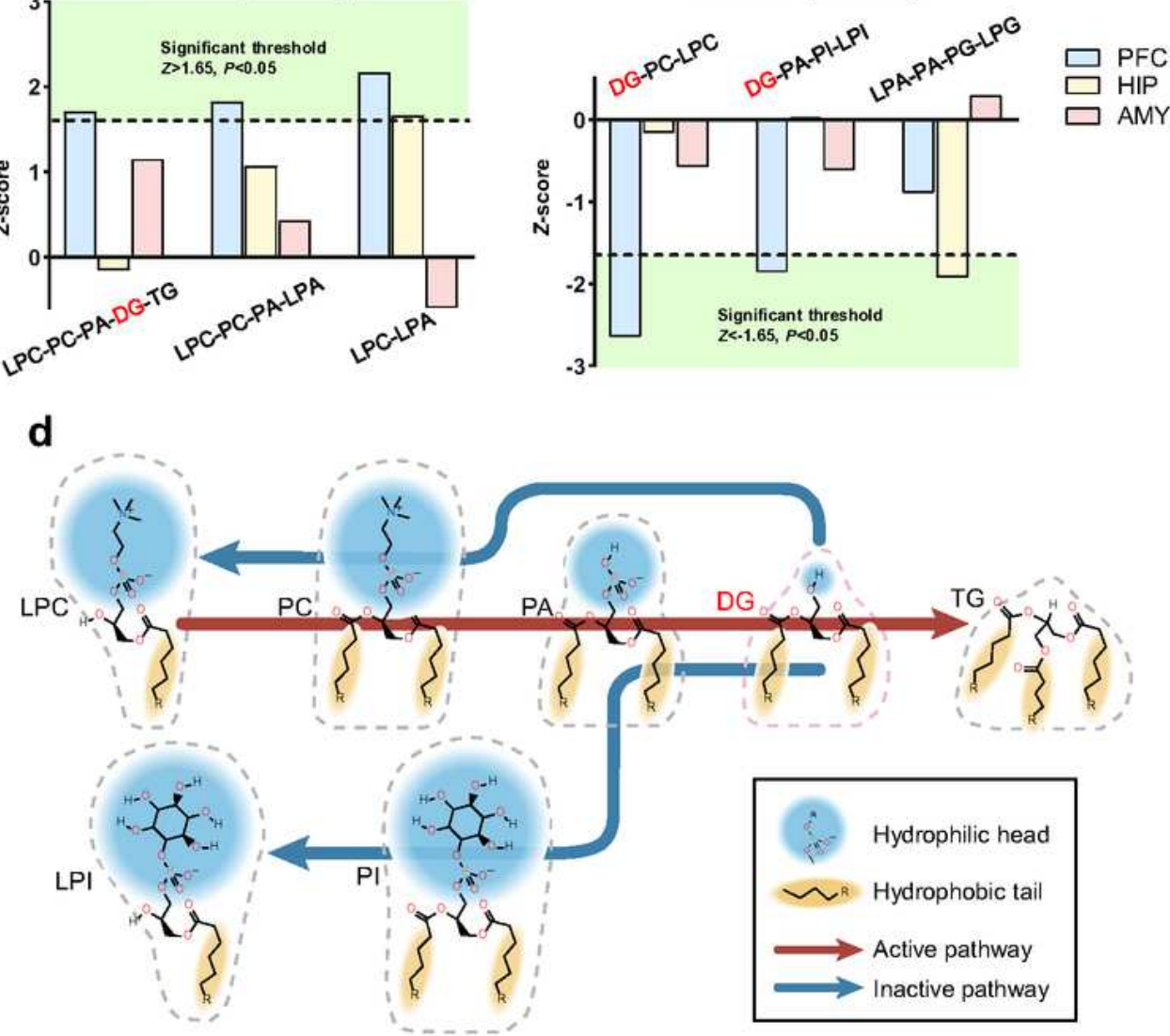

\section{Figure 4}

DG-related lipid pathway activity was particularly altered in the PFC of DL macaque. Analysis of lipid pathway activity showed altered predicted lipid fluxes occurred in brain regions. Calculated Z-scores were chosen to indicate the active/inactive pathways in DL relative to $\mathrm{HC}$, with the positive/negative values.(a) The PFC of DL macaque showed major altered lipid pathways, which were 3 active pathways LPC-PC-PADG-TG, LPC-PC-PA-LPA and LPC-LPA, and 2 inactive pathway DG-PC-LPC, DG-PA-PI-LPI, respectively. 
Altered lipid pathway activity was calculated by Z-score of each lipid classes in $\mathrm{DL}(\mathrm{n}=6)$ relative to $\mathrm{HC}(\mathrm{n}=6)$. Red and blue arrows show reactions with positive and negative activity, respectively. Colored circles indicate the fold changes in lipid abundance between 2 groups (-0.47-1.0). Deep arrows indicate the shared lipid flux across the network. (b-c) The HIP of DL macaque showed minor altered lipid pathways, including active pathway LPC-LPA and inactive pathway LPA-PA-PG-LPG. (d) molecular geometric alteration of DG related lipid pathways. Blue and yellow backgrounds indicated head groups and hydrophobic part. Dashed frames showed the geometry of lipids molecule, which can be defined as cone (DG, PA, TG), cylinder (PC and PI) and inverted cone (LPI and LPC).

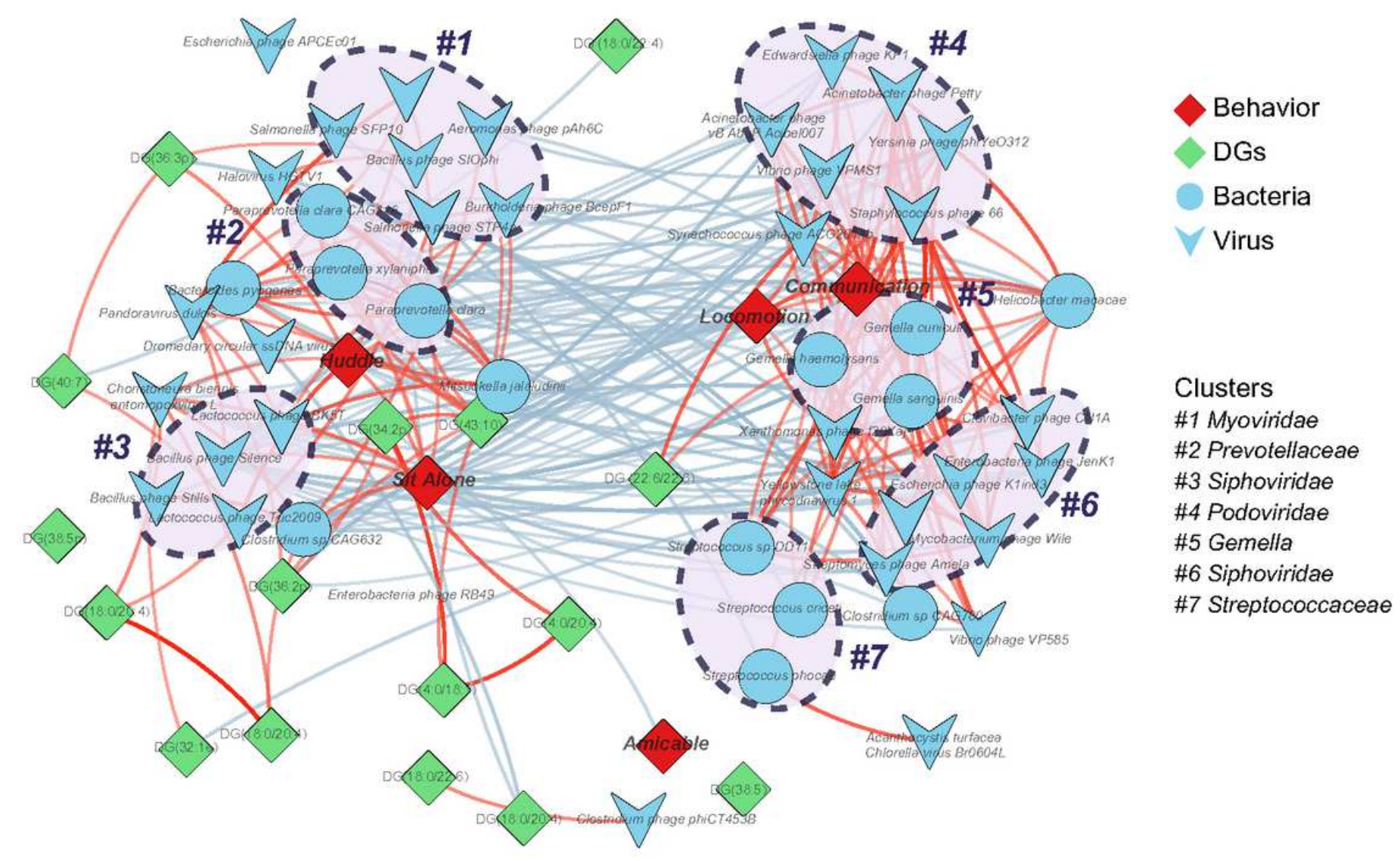

Figure 5

Network analyses of changed microbiome, DG in PFC and host phenotype interactions. The microbial species changed in DL were identified by LDA (LDA > 2), including 14 bacterial and 33 viral species. Hostmicrobiota interaction network was built from Spearman's non-parametric rank correlation coefficient $(\mathrm{P}<$ 0.05 ), and showed with coefficient $>0.70$ or $<-0.70$. Blue circles and $V$ triangles indicate the altered bacteria and virus respectively, green rhombus indicate the DG species in PFC and red rhombus indicated the depressive behaviors. Edge-weighted Spring-Embedded Layout was used to cluster nodes in 
accordance with coefficient. In result, there were 3 clusters correlated with the behaviors that represent negative emotions and unhealthy effects (Huddle and Sit alone), mainly focused on bacterial family Prevotellaceae and viral families Myoviridae and Siphoviridae. There were 4 clusters correlated with behaviors that represent positive and healthy emotions (Locomotion and Communication), mainly focused on bacterial families Gemella and Streptococcaceae and viral families Podoviridae and Siphoviridae. No clusters correlated with Amicable behavior that represents positive and healthy emotions. Red and blue lines indicate the correlation coefficient and the color depth was consistent with the coefficient $(-0.70-0.70)$.

\section{Supplementary Files}

This is a list of supplementary files associated with this preprint. Click to download.

- Additionfile1.docx

- Additionfile2.docx

- Additionfile3.docx

- Additionfile4.docx

- workflow.pdf 